\title{
RESISTANCE-MEASUREMENT STUDIES OF THE PECULIARITIES OF THE STRUCTURAL STATE OF CHROMIUM-NICKEL-MOLYBDENUM AUSTENITIC REACTOR STEELS DOPED WITH TITANIUM AND PHOSPHORUS
}

\author{
V. L. Arbuzov, V. I. Bobrovskii ${ }^{*}$, S. E. Danilov, V. V. Sagaradze \\ M.N. Miheev Institute of Metal Physics, Ural Branch of the Russian Academy of Sciences, \\ Ekaterinburg, Russian Federation \\ *Corresponding author. E-mail: bobrovskii@imp.uran.ru \\ Address for correspondence: ul. S. Kovalevskoy, 18, 620990, Ekaterinburg, Russian Federation \\ Tel: +7 (343) 3740003
}

Understanding of the mechanisms of radiation-induced phenomena is of fundamental value both for design of new steels and for improvement of the properties of the existing ones. Along with the crystal structure and chemical composition of the matrix, doping admixtures and the material microstructure play important role in these phenomena. In this paper, the peculiarities of the microstructural state of chromium-nickel-molybdenum austenitic steels doped with titanium and phosphorus are studied by means of resistance measurements. The existence of the synergetic effects of simultaneous doping of steels with titanium and phosphorus has been confirmed. The effect of solid solution segregation as the annealing temperature reaches 700-800 K, accompanied by increasing electric resistivity, and has been found. The energies of the migration of interstitial atoms and of the dissociation of the "interstitial site - titanium atom" pair have been determined. The phenomena observed are in agreement with the results obtained for other austenitic steels.

Keywords: austenitic reactor steels, segregation, intermetallics, doping, electrical resistance.

DOI: $10.17804 / 2410-9908.2017 .6 .091-102$

\section{References}

1. Zeman A., Kaiser R., Inozemtsev V., Beatty R.L. IAEA activities on coordinated research of structural materials for advanced reactor systems. J. Nucl. Mater., 2012, vol. 428, pp. 3-5. DOI: 10.1016/j.jnucmat.2012.06.024

2. Voyevodin V.N., Neklyudov I.M. Problems of radiation stability of structural materials for nuclear power industry. Visnyk Kharkivskogo Universitetu, ser. fiz., 2006, vol. 746, no. 4, pp. 3-22. (In Russian).

3. Was G.S. Fundamentals of Radiation Materials Science. Metals and Alloys. New York, Springer Science+Business Media, 2007, 1002 p.

4. Voronin V.I., Arbuzov V.L., Bobrovskii V.I., Danilov S.E., Kozlov K.A., Proskurnina N.V., Sagaradze V.V. Peculiarities of radiation-induced processes in the Cr-Ni-Mo austenitic steels studied by neutron diffraction. Diagnostics, Resource and Mechanics of materials and structures, 2015, iss. 5, pp. 80-89. Available at: URL: http://dream-journal.org/issues/2015-5/2015-5_46.html (accessed 20.11.2017).

5. $\quad$ Sagaradze V.V., Nalesnik V.M., Lapin S.S., Aliabev V.M. Precipitation hardening and radiation damageability of austenitic stainless steels. J. Nucl. Mater., 1993, vol. 202, pp.137-144. DOI: 10.1016/0022-3115(93)90036-X

6. Okita T., Wolfer W.G., Garner F.A., Sekimura N. Effects of titanium additions to austenitic ternary alloys on microstructural evolution and void swelling. Philosophical Magazine, 2005, vol. 85, no. 18, pp. 2033-2048. DOI: 10.1080/14786430412331331871

7. Maziasz P.J. Formation and stability of radiation-induced phases in neutron irradiated austenitic and ferritic steels. J. Nucl. Mater., 1989, vol. 169, pp. 95-115. DOI: 10.1016/0022-3115(89)90525-4

Arbuzov V.L. et al. / Resistance-measurement studies of the peculiarities of the structural state of chromium-nickel-molybdenum austenitic reactor steels doped with titanium and phosphorus 
8. Kurishita H., Muroga T., Watanabe H., Yoshida N., Kayano H., Hamilton M.L. Effect of FFTF irradiation on tensile properties of P- and Ti-modified model austenitic alloys with small amounts of boron. J. Nucl. Mater., 1994, vol. 212, pp. 519-524. DOI: 10.1016/0022-3115(94)90115-5

9. Shibahara I., Akasaka N., Onose S., Okada H., Ukai S. Swellling of advanced austenitic stainless steels developed for the environment of heavy neutron exposure. J. Nucl. Mater., 1994, vol. 212-215, pp. 487-491. DOI: 10.1016/0022-3115(94)90109-0

10. Brailsford A.D., Mansur L.K. The effect of precipitate-matrix interface sinks on the growth of voids in the matrix. J. Nucl. Mater., 1981, vol. 104, pp. 1403-1408. DOI: 10.1016/0022-3115(82)90796-6

11. Garner F.A., Wolfer W.G. The effect of solute additions on void nucleation. J. Nucl. Mater., 1981, vol. 102, pp. 143-150. DOI: 10.1016/0022-3115(81)90554-7

12. Watanabe H., Aoki A., Murakami H., Muroga T., Yoshida N. Effects of phosphorus on defect behavior, solute segregation and void swelling in electron irradiated Fe-Cr-Ni alloys. J. Nucl. Mater., 1988, vol. 155-157, pp. 815-822. DOI: 10.1016/0022-3115(88)90422-9

13. Watanabe H., Muroga T., Yoshida N. The temperature dependent role of phosphorus and titanium in microstructural evolution of Fe-Cr-Ni alloys irradiated in FFTF. J. Nucl. Mater., 1996, vol. 228, pp. 261-274. DOI: 10.1016/0022-3115(96)80004-3

14. Arbuzov V.L., Danilov S.E. Effect of titanium doping on accumulation and annealing of radiation defects in austenitic steel $16 \mathrm{Cr} 15 \mathrm{Ni} 3 \mathrm{Mo}(0-1) \mathrm{Ti}$ at low temperature $(80 \mathrm{~K})$ electron irradiation. In: IOP Conf., series: Materials Science and Engineering, 2016, vol. 110, pp. 01203310120335. DOI: 10.1088/1757-899X/110/1/012033

15. Danilov S.E., Arbuzov V.L., Kazantsev V.A. Radiation-induced separation of solid solution in Fe-Ni invar. J. Nucl. Mater., 2011, vol. 414, pp. 200-204. DOI: 10.1016/j.jnucmat.2011.03.013

16. Alyab'yev V.M., Vologin V.G., Dubinin S.F., Lapin S.S., Parkhomenko V.D., Sagaradze V.V. Neutron diffraction and electron microscopic investigation of decomposition and radiationinduced ageing of Cr-Ni-Ti austenitic alloys. Physics of Metals and Metallography, 1990, vol. 70, no. 2, pp. 131-137.

17. Arbuzov V.L., Shalnov K.V., Danilov S.E., Davletshin A.E., Pecherkina N.L., Sagaradze V.V. Observation of segregation deposits in iron-nickel-titanium alloy using scanning tunneling microscopy. Technical Physics Letters, 1999, vol. 25, no. 2, pp. 134-135. DOI: 10.1134/1.1262377

18. Houdremont E. Handbuch der Sonderstahlkunde. Berlin-Gottingen-Heidelberg, SpringerVerlag, 1956, $1038 \mathrm{p}$.

19. Arbuzov V.L., Danilov S.E., Kazantsev V.A., Sagaradze V.V. Radiation-induced strengthening of Al- and Ti-modified Fe-Ni alloys during electron irradiation. Physics of Metals and Metallography, 2011, vol. 115, pp. 1017-1022. DOI: 10.1134/S0031918X14100032

20. Hironobu A., Kuramoto E. Recovery of electrical resistivity of high-purity iron irradiated with 30 МэB electrons at 77 K. J. Nucl. Mater., 2000, vol, 283-287, pp. 174-178. DOI: $10.1016 / \mathrm{S} 0022-3115(00) 00339-1$ 
Подана в журнал: 21.11 .2017

УДК 669.15-194.56:620.183.6:539.2:620.181

DOI: $10.17804 / 2410-9908.2017 .6 .091-102$

\title{
РЕЗИСТИВНЫЕ ИССЛЕДОВАНИЯ ОСОБЕННОСТЕЙ СТРУКТУРНОГО СОСТОЯНИЯ ХРОМ-НИКЕЛЬ-МОЛИБДЕНОВЫХ АУСТЕНИТНЫХ РЕАКТОРНЫХ СТАЛЕЙ, ЛЕГИРОВАННЫХ ТИТАНОМ И ФОСФОРОМ
}

\author{
В. Л. Арбузов, В. И. Бобровский, С. Е. Данилов, В. В. Сагарадзе \\ Федеральное государственное бюджетное учреждение науки \\ Институт физики металлов имени М. Н. Михеева Уральского отделения Российской академии наук, \\ Екатеринбург, Российская Федераџия
}

\author{
*Ответственный автор. Электронная почта: bobrovskii@imp.uran.ru \\ Адрес для переписки: 620990 Екатеринбург, ул. С. Ковалевской, 18, Российская Федерация \\ Тел.: 8(343)374-00-03
}

Понимание механизмов радиационно-индуцированных явлений имеет фундаментальное значение для разработки новых и улучшения свойств существующих реакторных сталей. В этих явлениях помимо кристаллической структуры и химического состава матрицы важнейшую роль играют легирующие примеси и микроструктурное состояние материалов. В работе средствами резистометрии изучены особенности микроструктурного состояния легированных титаном и фосфором хром-никель-молибденовых аустенитных сталей. Подтверждено существование синергических эффектов одновременного легирования стали титаном и фосфором. Выявлен эффект расслоения твердого раствора при повышении температуры отжига до 700-800 K, сопровождающегося ростом электросопротивления. Установлены энергии миграции собственных межузельных атомов, а также диссоциации комплексов собственное междоузлие - атом титана. Наблюдавшиеся эффекты согласуются с результатами, полученными для других аустенитных сталей.

Ключевые слова: аустенитные реакторные стали, расслоение, интерметаллиды, легирование, электросопротивление.

\section{1. Введение}

Разработка новых конструкционных материалов для ядерной техники, удовлетворяющих требованиям безопасности и экономической эффективности, представляет собой исключительно важную и серьезную проблему. Определение направлений поиска создания новых перспективных материалов и методов улучшения свойств уже известных, требует понимания фундаментальных механизмов идущих под облучением процессов, многие аспекты которых изучены совершенно недостаточно. Как следствие этого, исследование радиационных повреждений в реакторных сталях различного типа продолжает оставаться весьма актуальной мировой тематикой [1-4]. В настоящее время в качестве основных конструкционных материалов для реакторов на быстрых нейтронах и термоядерных реакторов используются различные типы сталей. Но наиболее употребительными в качестве материала для тепловыделяющих сборок остаются аустенитные стали, как в силу их высоких механических свойств, так и хорошей технологичности. Однако, хотя на сталях этого типа на быстрых реакторах достигнуты неплохие эксплуатационные показатели, в частности уровень выдерживаемых повреждающих доз поднят примерно до 100 сна (смещений на атом), для высокой конкурентоспособности этой отрасли энергетики в дальнейшем потребуются уровни не менее 150 сна. Соответственно в мире сохраняется высокий интерес к поиску дальнейших спо-

Arbuzov V.L. et al. / Resistance-measurement studies of the peculiarities of the structural state of chromium-nickel-molybdenum austenitic reactor steels doped with titanium and phosphorus 
собов улучшения их свойств и поэтому объектом наших исследований выбраны аустенитные стали.

Основное препятствие в решении этой проблемы представляет склонность аустенитных сталей к вакансионному распуханию при высокодозовом облучении. Основные методы повышения радиационной стойкости лежат в области увеличения концентрации стоков точечных дефектов, в частности легирование различными химическими элементами, а также термомеханические обработки. Наши исследования нацелены на изучение эффектов, развивающихся при легировании таких материалов двумя примесями - титаном и фосфором.

Известно, что добавки $(0,2-1,0)$ мас. \% кремния или титана в аустенитные стали приводят к существенному снижению вакансионного распухания благодаря образованию дисперсных выделений $\gamma$-фазы [5]-[7]. Однако также было установлено [6], что такое воздействие добавок титана хотя и ослабляет процесс зарождения пор, но зато сопровождается повышением скорости роста сформировавшихся пор и вообще существенно ослабляется при высоких температурах облучения.

Подтверждено, что добавка в аустенитные стали фосфора приводит к выделению в них фосфидов $[8,9]$, также способных играть роль прямых и косвенных стоков точечных дефектов. Однако по поводу механизма этого явления высказываются различные мнения [10-12]. По-видимому, влияние фосфора на сдерживание распухания обусловлено несколькими причинами и зависит не только от условий облучения, но и от наличия других добавок. В частности высказывалось предположение, что стабильность и эффективность фосфидов может быть повышена за счет добавления титана [13]. Подтверждение существования синергических эффектов двойного легирования является одной из основных задач нашего исследования.

Конкретной целью этих экспериментов было с помощью резистивных методов исследования отследить возможные тонкие изменения микроструктуры материала и особенности поведения радиационных дефектов в нем при описанном легировании, а также при термообработках.

\section{2. Материал и методика}

Объектом наших исследований выбраны аустенитные реакторные стали типа X16H15М3. Химический состав стали: $\mathrm{Cr}-16 \%$; $\mathrm{Ni}-15 \%$; $\mathrm{Mo}-2,5 \%$; $\mathrm{Si}-0,5 \% ; \mathrm{Mn}-0,38 \% ; \mathrm{C}-0,03 \%$; $\mathrm{Fe}$ - остальное; остаточный $\mathrm{P}-0,005 \%$.

Образцы для исследований были выплавлены в вакуумной индукционной печи. Состав исследованных сплавов по титану и фосфору приведен в таблице.

Составы исследованных сплавов (мас. \%) по Ті и Р

\begin{tabular}{|c|c|c|c|c|c|c|c|}
\hline $\mathrm{Ti}$ & - & - & - & 0,6 & 1,0 & 1,0 & 1,0 \\
\hline $\mathrm{P}$ & 0,005 & 0,060 & 0,100 & 0,005 & 0,005 & 0,060 & 0,100 \\
\hline
\end{tabular}

Для электрофизических исследований образцы были прокатаны до толщины 150-200 мкм с последующей резкой на электроискровом станке. Полученные образцы были подвергнуты отжигу при температуре $1323 \mathrm{~K}$ в течение 30 мин с последующей закалкой в воду. Размеры зерна после обработки составляли 100-200 мкм, а плотность дислокаций около $10^{7} \mathrm{~cm}^{-2}$. Рентгеноструктурный и электронно-микроскопический анализ подтвердили однофазность полученных аустенитных сталей в закаленном состоянии. Часть закаленных образцов была подвергнута отжигам при различных температурах.

Облучение образцов нейтронами проводили в реакторе ИВВ-2М при 77-300 К в интервале флюенсов $(1,5-15) \cdot 10^{18} \mathrm{~cm}^{-2}$. Облучение образцов электронами с энергией 5 МэВ проводилось на линейном ускорителе ЛУЭ 5 при температурах 80-290 К до флюенсов $(1-4) \cdot 10^{18} \mathrm{~cm}^{-2}$.

Arbuzov V.L. et al. / Resistance-measurement studies of the peculiarities of the structural state of chromium-nickel-molybdenum austenitic reactor steels doped with titanium and phosphorus 
Измерения остаточного электросопротивления (при 4,2 К) выполнялись с использованием стандартного четырехконтактного метода с чувствительностью на уровне 0,01 нОм·см и точностью $0,02 \%$ [14]. Измерение остаточного электросопротивления чувствительный, точный и экспрессный метод позволяющий отслеживать структурные изменения в металлах и сплавах, следить за изменениями в концентрациях точечных дефектов на уровне ppm. Однако этот метод является интегральным, и для правильной интерпретации полученных данных, необходимо знать какие процессы оказывают влияние на наблюдаемые изменения.

\section{3. Результаты и обсуждение}

Для проявления эффектов легирования материала в сочетании с термическими воздействиями образцы аустенитной стали с минимальным содержанием фосфора и концентрациями титана 0, 0,6 и 1,0 мас. \% были подвергнуты гомогенезирующему отжигу при температуре $1323 \mathrm{~K}$ в течение 30 мин, с последующей закалкой в воду. Затем часть их была отожжена при $923 \mathrm{~K}$ в течение 5 ч для формирования $\gamma^{\prime}$-фазы, присутствие которой в виде выделений размером 7-10 нм с концентрацией (4.6) $10^{16} \mathrm{~cm}^{-3}$ было подтверждено с помощью электронной микроскопии и сканирующей туннельной микроскопии. Другая часть образцов была подвергнута отжигу при $773 \mathrm{~K}$ в течение 3 ч с целью формирования расслоения твердого раствора, как это наблюдалось в [15].

Прежде всего, было исследовано поведение остаточного электросопротивления образцов, взятых в изначальном закаленном состоянии, а затем подвергнутых серии последовательных изохронных отжигов. Относительное изменение остаточного электросопротивления в зависимости от температуры изохронного отжига (за базисный уровень принимали значения остаточного сопротивления закаленных образцов) представлено на рис. 1.

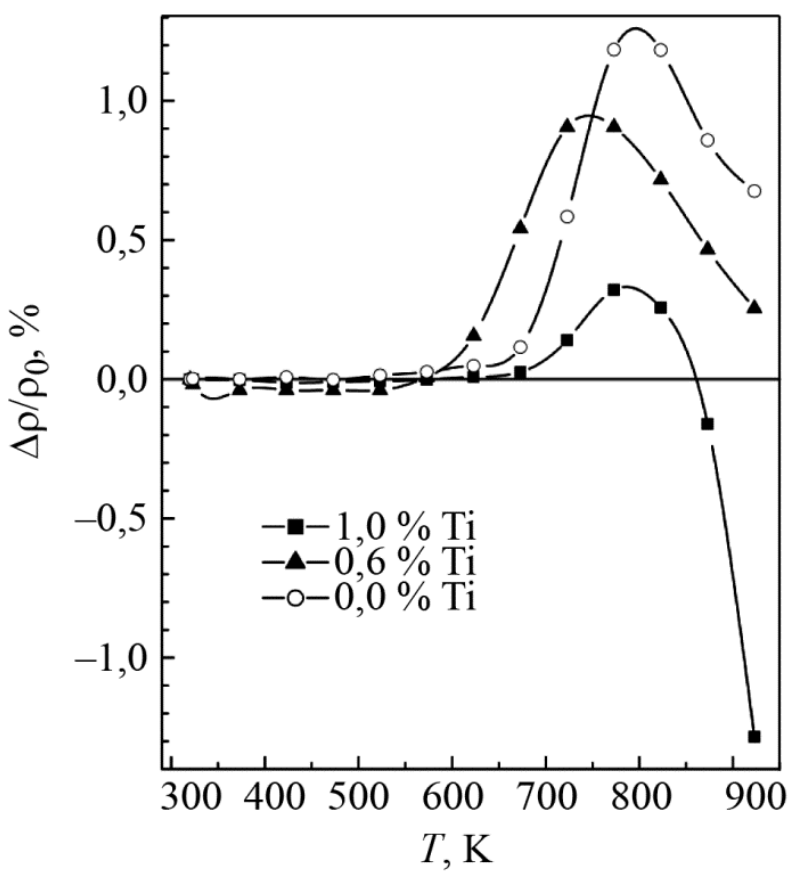

Рис. 1. Зависимость относительного изменения остаточного электросопротивления образцов, взятых в исходном закаленном состоянии, от температуры последующих изохронных отжигов

Независимо от содержания титана рост температуры отжига в интервале (700-800) K ведет к росту электросопротивления. Причем величина прироста относительно велика, что свидетельствует о структурных изменениях. При этом максимальный рост относительного 
электросопротивления наблюдается в сплаве без титана при 800 К, следовательно данный процесс не связан с образованием интерметаллида $\mathrm{Ni}_{3} \mathrm{Ti}$ при старении, что обычно приводит к уменьшению величины подобных пиков). Вероятно, данный эффект связан с атомным расслоением твердого раствора по никелю и хрому, который существенно сильнее влияет на электросопротивление, чем начальные этапы старения [16].

Дальнейшее повышение температуры изохронных отжигов приводит к падению электросопротивления. Наиболее ярко этот эффект проявляется для сплава с содержанием титана $1 \%$, где падение относительного сопротивления ниже исходной величины прямо указывает на уменьшение концентрации титана и никеля в твердом растворе в результате выделения $\mathrm{Ni}_{3} \mathrm{Ti}$. Тот факт, что максимум роста остаточного электросопротивления тем выше, чем ниже концентрация титана, свидетельствует о том, что легирование титаном оказывает подавляющее действие на этот процесс.

Старение образцов при $923 \mathrm{~K}$ приводит к выделению части титана в виде интерметаллидных частиц типа $\mathrm{Ni}_{3} \mathrm{Ti}$, что было подтверждено в наших предшествующих исследованиях титансодержащих $\mathrm{Fe}-\mathrm{Ni}$ сплавов [17]. Исследования поведения остаточного сопротивления этих образцов показали такой же его рост в интервале температур отжига 700-800 K с последующим падением, причем с той же зависимостью от концентрации титана (рис. 1).

Как выяснилось, легирование фосфором оказывает существенное воздействие на наблюдаемые в этих сталях эффекты. Известно [18], что растворимость фосфора в аустенитных сплавах на основе железа гораздо меньше, чем растворимость фосфора в $\alpha$-фазе железа, доходящей при $500{ }^{\circ} \mathrm{C}$ до $0,25 \%$. С учетом фазовой диаграммы железо-фосфор, можно ожидать в наших образцах наличия фосфидов $\mathrm{Fe}_{3} \mathrm{P}$ и $\mathrm{Fe}_{2} \mathrm{P}$. Действительно, электронная микроскопия подтвердила наличие в наших легированных фосфором образцах малого количества дисперсных игольчатых частиц, которые вероятнее всего являются фосфидами железа. При нейтронографической аттестации образцов аустенитной стали состава X16H15M3T1 с добавками фосфора $(0,005 ; 0,06 ; 0,1)$ мас. \%. набор рефлексов на всех нейтронограммах соответствовал аустенитной ГЦК-решетке. Дополнительных рефлексов интенсивностью более 0,2 \% от интенсивности основных рефлексов обнаружено не было (рис. 2).

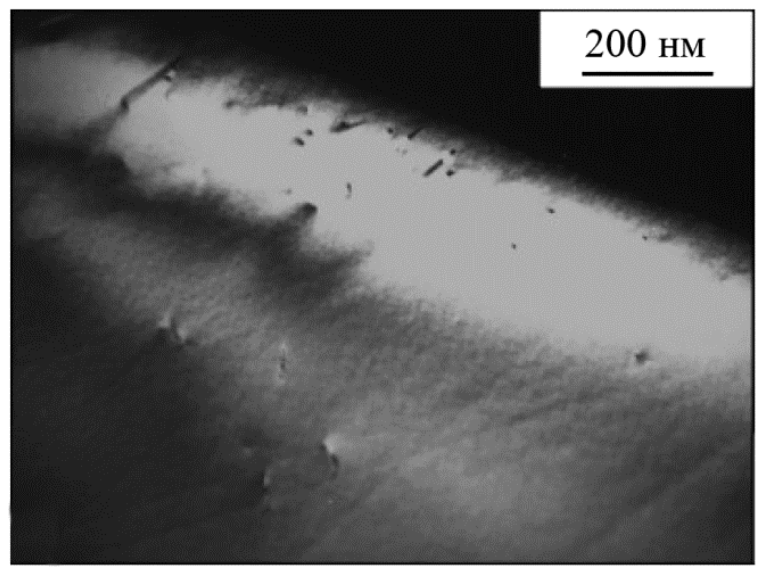

Рис. 2. Микроструктура сплава Х16Н15М3 с 0,06 мас. \% фосфора после закалки от 1373 К (выдержка 20 мин) и старения при 673 К (выдержка 24 ч). Наблюдаются игольчатые выделения фосфидов

На рис. 3 представлены кривые относительного изменения относительного остаточного электросопротивления в зависимости от температуры изохронного отжига образцов стали Х16Н15М3 и Х16Н15М3Т1, дополнительно легированных 0,06 и 0,1 мас. \% фосфора, взятых, как и образцы на рис. 1, изначально в закаленном состоянии. 


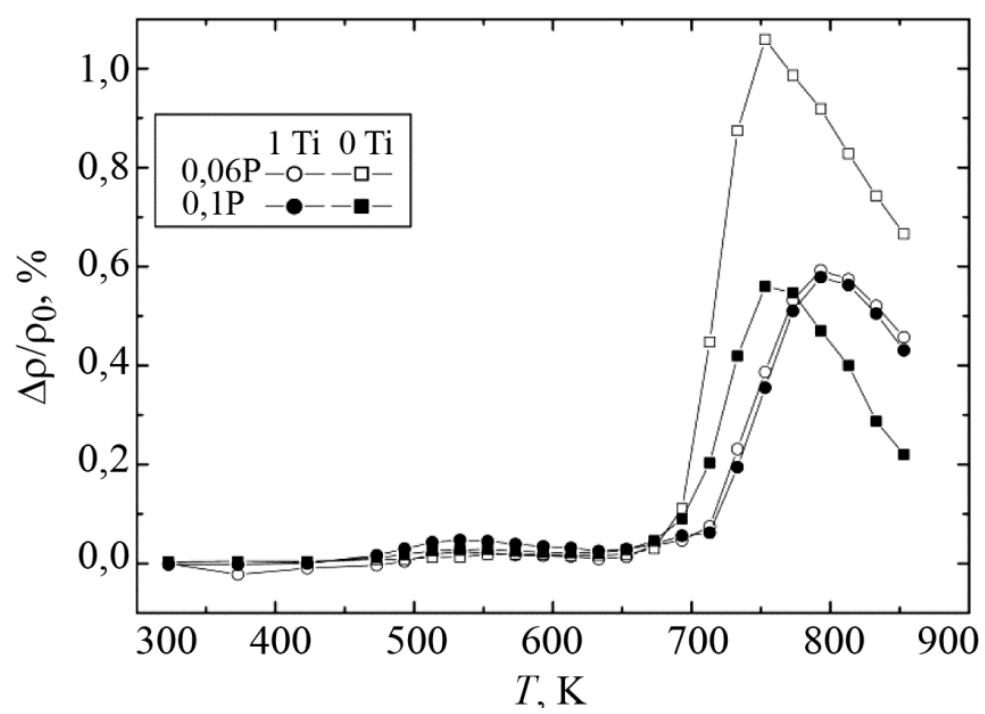

Рис. 3. Зависимость относительного изменения остаточного электросопротивления образцов стали Х16Н15М3 с различным легированием по титану и фосфору, взятых в исходном

закаленном состоянии, от температуры последовательных изохронных отжигов

На обоих рис. 1 и 3 наблюдаются те же процессы роста остаточного электросопротивления после отжигов при 700-800 К и его снижения при температурах 850-950 К.

Обратим внимание, что рост относительного электросопротивления в интервале (700-800) К и его снижение в интервале (850-950) К наблюдается как на сталях, легированных титаном, так и на сталях без титана, причем присутствие титана, как и на рис. 1, ослабляет этот процесс. Такие же изменения сопротивления при повышении температуры и подавление процесса в случае легирования титаном наблюдались в процессе расслоения твердого раствора в инварных ГЦК сплавах типа $\mathrm{Fe}-\mathrm{Ni}$. Отметим, что в сплаве $\mathrm{Fe}-\mathrm{Ni}-\mathrm{Ti}$, присутствие $5 \%$ титана полностью подавляет процесс расслоения [19]. Это дает основания считать, что наблюдаемые в нашем случае изменения электросопротивления при (700-800) К также связаны с процессами расслоения твердого раствора. Повышение температуры отжига выше $800 \mathrm{~K}$ в наших образцах приводит к гомогенизации твердого раствора и понижению остаточного электросопротивления в соответствии с изменениями равновесного состояния твердого раствора. Легирование титаном подавляет процесс расслоения твердого раствора.

Отметим, что без легирования титаном пока содержание фосфора остается низким 0,06 мас. \%, электросопротивление образцов стали без легирования титаном показывает то же поведение, что и практически бесфосфорная сталь, демонстрируя столь же высокий максимум роста (выше $1 \%$ ), при этом легирование стали всего лишь $0,1 \%$ фосфора подавляет этот рост уже до $0,6 \%$. В то же время в случае сплава, легированного $1 \%$ титана, влияние добавки к нему 0,1 \% фосфора вообще не выявляется, что подтверждает наличие синергических эффектов при одновременном легировании аустенитных сталей титаном и фосфором.

Наиболее важным для нас вопросом является выяснение механизма воздействия легирования этих сталей на протекание в них радиационно-индуцированных процессов. На данном этапе наши радиационные исследования пока ограничились только образцами, легированными титаном. Весьма удобным методом изучения поведения радиационно-индуцированных точечных дефектов является низкотемпературное облучение материалов электронами, сопровождаемое серией последующих изохронных отжигов, в процессе которых температура материала пробегает ряд значений от температуры облучения до максимальной температуры. С помощью такой методики удается разделять по шкале энергий сложные радиационно-индуцированные процессы, которые при других условиях облучения и отжига протекают единовременно и, следовательно, трудноразличимы.

Arbuzov V.L. et al. / Resistance-measurement studies of the peculiarities of the structural state of chromium-nickel-molybdenum austenitic reactor steels doped with titanium and phosphorus 
Вакансионные дефекты (вакансии и вакансионные кластеры - ВК) играют большую роль в радиационно-индуцированных структурно-фазовых превращениях. При бескаскадном электронном облучении ВК образуются в процессе свободной миграции вакансий и их взаимодействия между собой. При каскадном нейтронном облучении они образуются непосредственно в каскадах смещений, причем их образование осложняется перекрытием каскадов. При повышении температуры после облучения, диссоциация ВК превращает их в источник свободно мигрирующих вакансий. Каскады атомных смещений усложняют картину радиационных повреждений в связи с тем, что ВК являются эффективными стоками точечных дефектов и влияют на диффузионную длину свободной миграции дефектов. Сравнение воздействия каскадного нейтронного облучения и бескаскадного электронного облучения весьма полезно для выявления роли свободно мигрирующих радиационных дефектов и каскадов атомных смещений.

В ходе исследований было установлено, что накопление радиационных дефектов при облучении электронами около $80 \mathrm{~K}$ ведет к линейному росту электросопротивления с дозой облучения, что соответствует росту концентрации сохраненных точечных дефектов. При этом выяснилось, что в исследуемых сплавах имеет место миграция собственных межузельных атомов с энергией активации 0,36 эВ к стокам и формирование комплексов собственное междоузлие - атом титана, проявляющиеся в температурном интервале (110-120) К. Диссоциация этих комплексов происходит около $170 \mathrm{~K}$. Энергия связи комплексов - 0,19 эВ.

Миграция вакансий возникает при (220-240) К с активационной энергией $(0,65$ $0,70)$ эВ. Это ведет к формированию вакансионных кластеров (ВК) и комплексов вакансия - атом титана. Диссоциация этих комплексов и вакансионных кластеров начинается в районе комнатной температуры и проявляется до $700 \mathrm{~K}$. Это ведет к радиационноиндуцированному расслоению твердого раствора, происходящему при пострадиационном изохронном отжиге.

Облучение образцов нейтронами проводили в реакторе ИВВ-2М при $77 \mathrm{~K}$ в интервале флюенсов $(1,5-15) \cdot 10^{18} \mathrm{~cm}^{-2}$. Наблюдавшийся при этом прирост относительного остаточного электросопротивления был существенно больше, чем при электронном облучении. Дозовые зависимости также претерпели качественные изменения и [27]. Отметим, что при дозах выше $1,510^{18} \mathrm{~cm}^{-2}$ происходит выход дозовых зависимостей на квазистационарный режим, что, вероятно, связано с ограничением роста концентрации радиационных дефектов при перекрытии каскадов атомных смещений.

На рис. 4 представлены зависимости изменений остаточного электросопротивления от температуры изохронного отжига образцов с $1 \% \mathrm{Ti}$, облученных нейтронами и электронами. В нижней части рис. 4 дифференцированные зависимости. Из графиков видно, что в отличие от электронного облучения, где наблюдались пики отжига при $110 \mathrm{~K}$ и $220 \mathrm{~K}$, при нейтронном облучении в диапазоне (80-250) K наблюдается более размытая структура с центром в районе $170 \mathrm{~K}$, что связано с каскадным характером облучения, при котором имеется широкий набор расстояний между собственными межузельными атомами и вакансиями.

Отметим, что в работе [20] было показано, что при облучении чистого железа при $77 \mathrm{~K}$ прирост электросопротивления линейно зависит от флюенса облучения как для каскадоообразующих облучений, так и для бескаскадного облучения, что означает, что прирост электросопротивления не зависит от того, распределены ли радиационные дефекты по объему образца гомогенно, или они находятся в областях с повышенной концентрацией вакансий в каскадах атомных смещений. Это дает нам основание полагать, что наблюдаемый нами прирост относительного электросопротивления отражает концентрацию радиационных дефектов, даже в случае перекрытия каскадов. 


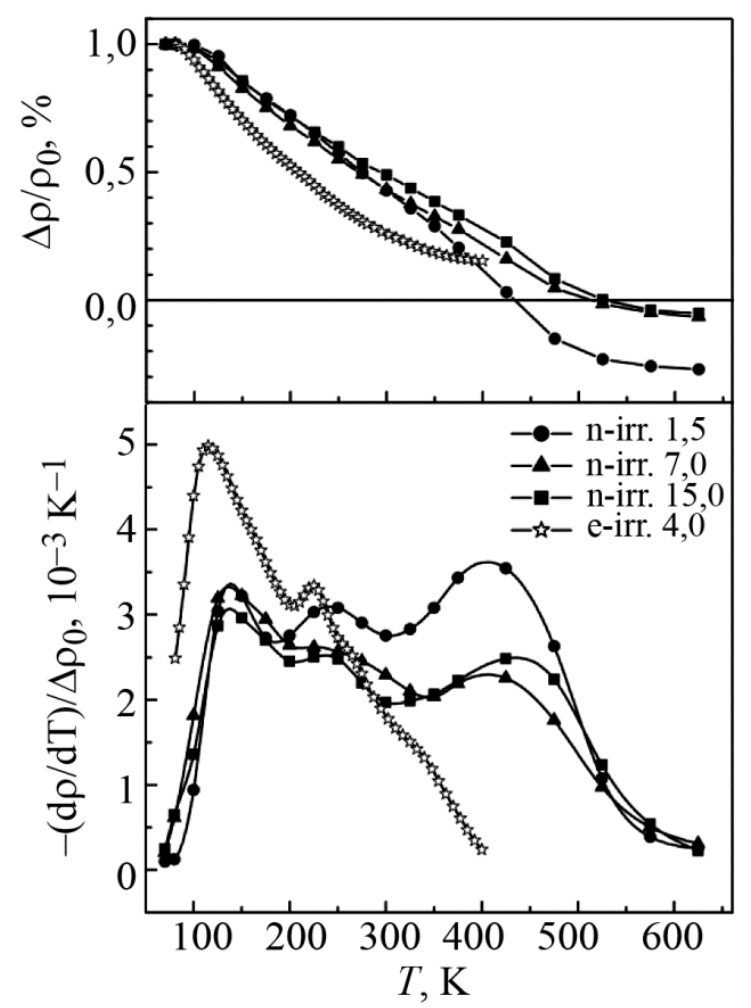

Рис. 4. Изменения остаточного электросопротивления при изохронном отжиге, нормированные на прирост остаточного электросопротивления после облучения при $80 \mathrm{~K}$ в стали 16Cr-15Ni-3Mo-1Ti: е - облучение электронами, флюенс $4 \cdot 10^{18} \mathrm{~cm}^{-2}$; $\mathrm{n}$ - облучение нейтронами, флюенсы $(1,5 ; 7,0 ; 15,0) \cdot 10^{18} \mathrm{~cm}^{-2}$

Пик отжига в районе $300 \mathrm{~K}$ характерен для обоих типов облучения. По-видимому, он связан с миграцией вакансий, образующихся при диссоциации наиболее мелких ВК, что дает возможность оценить энергию их диссоциации в $(1,0-1,1)$ эВ. Более сложные (крупные) ВК, образующиеся при миграции вакансий и ВК, образовавшиеся в каскадах атомных смещений, диссоциируют примерно при одной температуре - около $480 \mathrm{~K}$, несмотря на то, что размеры (кратность) этих кластеров существенно различаются. Оценки энергии диссоциации вакансионных кластеров дают $(1,4-1,5)$ эВ.

Отметим, что в работе [20] было показано, что при облучении чистого железа при $77 \mathrm{~K}$ прирост электросопротивления линейно зависит от флюенса облучения как для каскадоообразующих облучений, так и для бескаскадного облучения, что означает, что прирост электросопротивления не зависит от того, распределены ли радиационные дефекты по объему образца гомогенно, или они находятся в областях с повышенной концентрацией вакансий в каскадах атомных смещений. Это дает нам основание полагать, что наблюдаемый нами прирост относительного электросопротивления отражает концентрацию радиационных дефектов даже в случае перекрытия каскадов.

На рис. 5 представлены зависимости прироста электросопротивления образцов стали Х16Н15М3, легированной 1 мас. \% титана при нейтронном и электронном облучениях при $77 \mathrm{~K}$ и отожженных после облучения при температурах 211 и $293 \mathrm{~K}$ в зав исимости от накопленной дозы облучения, выраженной в количестве смещений на атом (сна, dpa). 


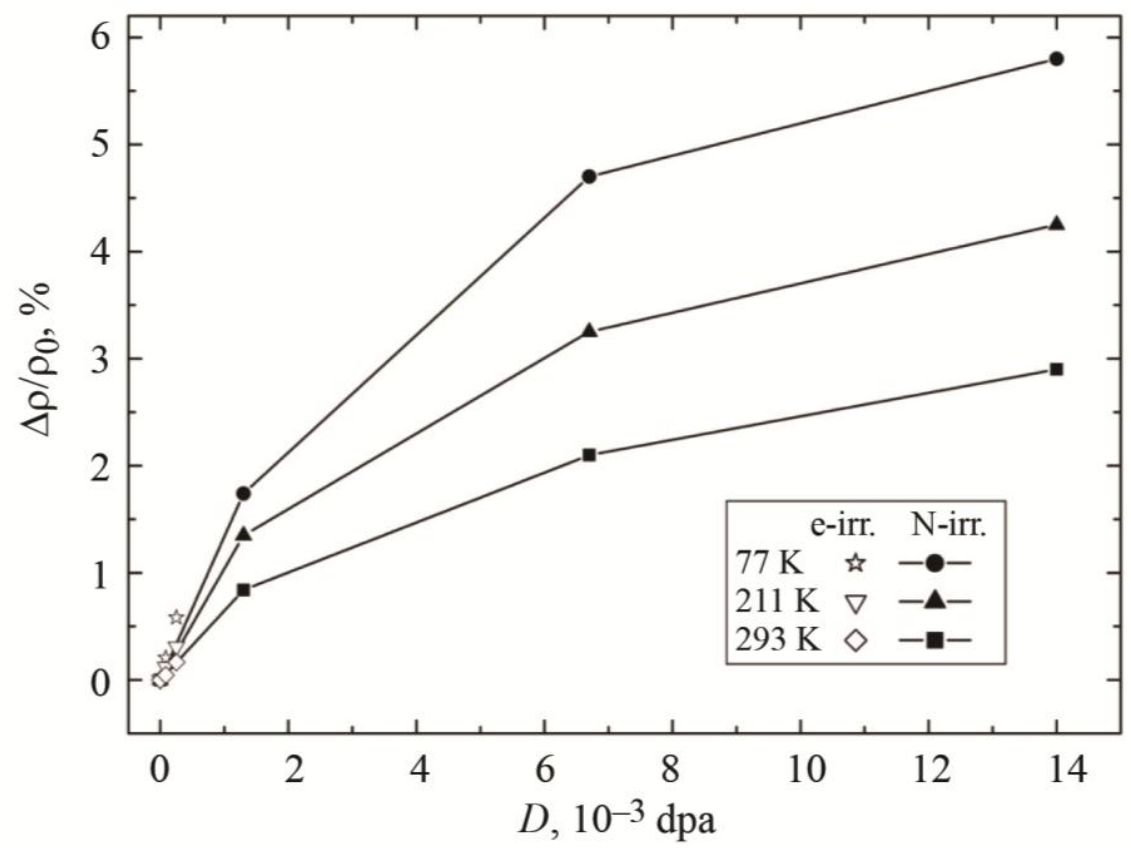

Рис. 5. Зависимость прироста относительного остаточного электросопротивления от величины повреждающей дозы $D$

Очевидно, что в диапазоне достигнутых доз результаты и электронного, и нейтронного облучений укладываются на общие зависимости. Отметим, что при дозе нейтронного облучения, большей $7 \cdot 10^{-3}$ сна, начинается перекрытие каскадов смещения, в связи с чем зависимости прироста электросопротивления от дозы приближаются к квазистационарному уровню, т. е. накопление радиационных дефектов практически прекращается. Отжиг радиационных дефектов при температурах 211 и 293 К происходит в среднем одинаково до перекрытия каскадов и после перекрытия каскадов.

\section{4. Заключение}

В результате резистивных исследований аустенитных реакторных сталей типа Х16Н15М3, легированных титаном и фосфором, получена информация об их микроструктуре, свойствах и радиационном поведении, обусловленном генерируемыми под облучением вакансиями, вакансионными кластерами и междоузлиями и их взаимодействиями с выделениями, созданными при легировании сталей. Подтверждено существование синергических эффектов одновременного легирования стали титаном и фосфором. Выявлен эффект расслоения твердого раствора при повышении температуры отжига до 700-800 К, сопровождающегося ростом электросопротивления. Это расслоение заметно подавляется при легировании титаном на уровне 1 мас. \% или фосфором на уровне 0,1 мас. \%. При дальнейшем повышении температуры отжига при 800-900 K происходит гомогенизация твердого раствора с уменьшением электросопротивления. В нержавеющих сталях типа X16H15M3 с различным содержанием титана $(0-1,0) \%$ расслоение твердого раствора без облучения заметно в районе 700 К. Облучение приводит к расслоению в районе температур 300-400 К за счет миграции вакансий, освобождающихся при диссоциации вакансионных кластеров. Этот процесс подавляется при увеличении концентрации титана, приводящего также к образованию частиц $\gamma^{\prime}$-фазы при отжигах выше 800 К. Установлены энергии миграции собственных межузельных атомов, а также диссоциации комплексов собственное междоузлие - атом титана. 


\section{Благодарность}

Работа выполнена с использованием УНУ «НМК ИФМ» при поддержке УрО РАН (проект № 15-17-2-3).

\section{Литература}

1. IAEA activities on coordinated research of structural materials for advanced reactor systems / A. Zeman, R. Kaiser, V. Inozemtsev, R. L. Beatty // J. Nucl. Mater. - 2012. - Vol. 428. - P. 3-5. DOI: 10.1016/j.jnucmat.2012.06.024

2. Воеводин В. Н., Неклюдов И. М. Проблемы радиационной стойкости конструкционных материалов ядерной энергетики // Вісник Харькивського университету. Сер. Физ. 2006. - T. 746, вып. 4. - C. 3-22.

3. Was G. S. Fundamentals of Radiation Materials Science. Metals and Alloys. - New-York : Springer Science+Business Media, 2007. - 1002 p.

4. Peculiarities of radiation-induced processes in the $\mathrm{Cr}-\mathrm{Ni}-\mathrm{Mo}$ austenitic steels studied by neutron diffraction / V. I. Voronin, V. L. Arbuzov, V. I. Bobrovskii, S. E. Danilov, K. A. Kozlov, N. V. Proskurnina, V. V. Sagaradze // Diagnostics, Resource and Mechanics of materials and structures. - 2015. - Iss. 5. - C. 80-89. - URL: http://dream-journal.org/issues/2015-5/2015-5_46.html (accessed 20.11.2017).

5. Precipitation hardening and radiation damageability of austenitic stainless steels / V. V. Sagaradze, V. M. Nalesnik, S. S. Lapin, V. M. Aliabev // J. Nucl. Mater. - 1993. - Vol. 202. - P.137-144. DOI: 10.1016/0022-3115(93)90036-X

6. Effects of titanium additions to austenitic ternary alloys on microstructural evolution and void swelling / T. Okita, W. G. Wolfer, F. A. Garner, N. Sekimura // Philosophical Magazine. 2005. - Vol. 85, no. 18. - P. 2033-2048. - DOI: 10.1080/14786430412331331871

7. Maziasz P. J. Formation and stability of radiation-induced phases in neutron irradiated austenitic and ferritic steels // J. Nucl. Mater. - 1989. - Vol. 169. - P. 95-115. - DOI: 10.1016/00223115(89)90525-4

8. Effect of FFTF irradiation on tensile properties of P- and Ti-modified model austenitic alloys with small amounts of boron / H. Kurishita, T. Muroga, H. Watanabe, N. Yoshida, H. Kayano, M. L. Hamilton // J. Nucl. Mater. - 1994. - Vol. 212. - P. 519-524. DOI: $10.1016 / 0022-3115(94) 90115-5$

9. Swellling of advanced austenitic stainless steels developed for the environment of heavy neutron exposure / I. Shibahara, N. Akasaka, S. Onose, H. Okada, S. Ukai // J. Nucl. Mater. - 1994. Vol. 212-215. - P. 487-491. - DOI: 10.1016/0022-3115(94)90109-0

10. Brailsford A. D., Mansur L. K. The effect of precipitate-matrix interface sinks on the growth of voids in the matrix // J. Nucl. Mater. - 1981. - Vol. 104. - P. 1403-1408. - DOI: 10.1016/00223115(82)90796-6

11. Garner F. A., Wolfer W. G. The effect of solute additions on void nucleation // J. Nucl. Mater. - 1981. - Vol. 102. - P. 143-150. - DOI: 10.1016/0022-3115(81)90554-7

12. Effects of phosphorus on defect behavior, solute segregation and void swelling in electron irradiated Fe-Cr-Ni alloys / H. Watanabe, A. Aoki, H. Murakami, T. Muroga, N. Yoshida // J. Nucl. Mater. - 1988. - Vol. 155-157. - P. 815-822. - DOI: 10.1016/0022-3115(88)90422-9

13. Watanabe H., Muroga T., Yoshida N. The temperature dependent role of phosphorus and titanium in microstructural evolution of Fe-Cr-Ni alloys irradiated in FFTF // J. Nucl. Mater. 1996. - Vol. 228. - P. 261-274. - DOI: 10.1016/0022-3115(96)80004-3

14. Arbuzov V. L., Danilov S. E. Effect of titanium doping on accumulation and annealing of radiation defects in austenitic steel $16 \mathrm{Cr} 15 \mathrm{Ni} 3 \mathrm{Mo}(0-1) \mathrm{Ti}$ at low temperature $(80 \mathrm{~K})$ electron irradiation // IOP Conf. Series: Materials Science and Engineering. - 2016. - Vol. 110. - P. 01203310120335. - DOI: 10.1088/1757-899X/110/1/012033 
15. Danilov S. E., Arbuzov V. L., Kazantsev V. A. Radiation-induced separation of solid solution in Fe-Ni invar // J. Nucl. Mater. - 2011. - Vol. 414. - P. 200-204. - DOI: 10.1016/j.jnucmat.2011.03.013

16. Neutron diffraction and electron microscopic investigation of decomposition and radiationinduced ageing of Cr-Ni-Ti austenitic alloys / V. M. Alyab'yev, V. G. Vologin, S. F. Dubinin, S. S. Lapin, V. D. Parkhomenko, V. V. Sagaradze // Physics of Metals and Metallography. - 1990. Vol. 70. - No. 2. - P. 131-137.

17. Observation of segregation deposits in iron-nickel-titanium alloy using scanning tunneling microscopy / V. L. Arbuzov, K. V. Shalnov, S. E. Danilov, A. E. Davletshin, N. L. Pecherkina, V. V. Sagaradze // Technical Physics Letters. - 1999. - Vol. 25. - No. 2. - P. 134-135. DOI: $10.1134 / 1.1262377$

18. Houdremont E. Handbuch der Sonderstahlkunde. - Berlin-Gottingen-Heidelberg : SpringerVerlag, 1956. - $1038 \mathrm{p}$.

19. Radiation-induced strengthening of Al- and Ti-modified Fe-Ni alloys during electron irradiation / V. L. Arbuzov, S. E. Danilov, V. A. Kazantsev, V. V. Sagaradze // Physics of Metals and Metallography. - 2011. - Vol. 115. - P. 1017-1022. - DOI: 10.1134/S0031918X14100032

20. Hironobu A., Kuramoto E. Recovery of electrical resistivity of high-purity iron irradiated with 30 MэB electrons at 77 K // J. Nucl. Mater. - 2000. - Vol. 283-287. - P. 174-178. DOI: 10.1016/S0022-3115(00)00339-1 